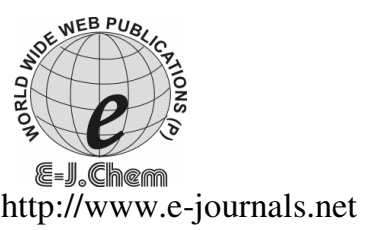

\title{
Polyaniline Conducting Electroactive Polymers: Thermal and Environmental Stability Studies
}

\author{
REZA ANSARI* and M. B. KEIVANI \\ *Chemistry Department, Faculty of science, \\ Guilan University, Rasht, Iran, POB 41335-1914. \\ Iranian Academic Center for Education, Culture \& Research (ACECR) \\ Guilan Branch, Rasht, Iran \\ E mail: ransari@guilan.ac.ir
}

Received 23 May 2006; Accepted 27 June 2006.

\begin{abstract}
In the current studies, polyaniline (PANi) was prepared both chemical and electrochemically in the presence of different bronsted acids from aqueous solutions. The effect of thermal treatment on electrical conductivity, and thermal stability of the PANi conducting polymers were investigated using 4-point probe and TGA techniques respectively. It was found that polymer prepared by $\mathrm{CV}$ method is more thermally stable than those prepared by the other electrochemical techniques. In this paper we have also reviewed some fundamental information about synthesis, general properties, diverse applications, thermal and environmental stability of polyaniline conducting polymers.
\end{abstract}

Keywords: Polyaniline, conducting polymer, electroactive, thermal and environmental stability

\section{Introduction}

Thermal studies of PANi conducting polymers are particularly important when one considers the use of elevated temperatures to process PANi and its blends into technologically useful forms. In any practical application, knowledge of the stability and degradation mechanism of this class of conducting polymers is also of primary importance. For many practical applications of polyaniline conducting polymers such as battery applications, the thermal stability of the material is very important. Among conducting polymers, a great deal of researches have been devoted to PANi due to its unique electrical, electrochemical properties, high environmental stability, easy polymerization and low cost of monomer ${ }^{1-10}$. 
Furthermore, the superior processibility of PANi stimulated several investigations for potential applications of this intrinsically conductive polymer. However, melt processing is not possible, since the polymer decomposes at temperatures below its softening or melting point. Polyaniline can be synthesised by the oxidative polymerisation of aniline in aqueous acidic media using a variety of oxidising agents such as $(\mathrm{NH})_{4} \mathrm{~S}_{2} \mathrm{O}_{8}, \mathrm{KIO}_{3}$ and $\mathrm{K}_{2} \mathrm{Cr}_{2} \mathrm{O}_{7}$. For large scale production the chemical method is more convenient because the scale of products in electrochemical synthesis depends on the size of electrode ${ }^{11-18}$. Polyaniline films can also be easily synthesised by electrochemical deposition employing potentiostatic, potentiodynamic or galvanostatic methods ${ }^{19-21}$ through oxidation of aniline in bronsted acid medium. A major problem however is the production of the conducting form of polyaniline. Therefore, it becomes necessary to critically control all the process parameters involved in a particular deposition technique so as to obtain the deposit only with the particular oxidation state of interest ${ }^{21}$. For example, cyclic potential sweep deposition produces films with better morphology, conductivity and structural integrity than potentiostatic deposition ${ }^{20}$.

Polymer synthesised using chemical methods is a powdery material and the properties of chemically synthesised polyaniline from aqueous solutions are determined by a wide variety of synthesis parameters, such as $p H$, aniline/oxidant ratios, polymerisation temperature and time, type/ concentration of oxidising agents and protonic acids ${ }^{14}$. It has been reported that the aniline/oxidant ratio has a minor effect on the electrical conductivity of the polyanilines produced. By contrast, the polymer yield was strongly dependent on this ratio. Polyaniline in its base form (by treatment with dilute aqueous alkali e.g. $0.1 \mathrm{M} \mathrm{NH} \mathrm{NH}_{4} \mathrm{OH}$ ) is insoluble in water and soluble in several aqueous and non-aqueous solvents such as concentrated sulphuric acid, acetic acid (\%80), formic acid, dimethylsulfoxide (DMSO), dimethylformamide (DMF), and 1-methyl 2-pyrrolidinone (NMP) ${ }^{15}$.

Large flexible films of emeralidine base can be cast from NMP solution which can be thermally stretch-aligned at $\sim 140{ }^{\circ} \mathrm{C}$ up to 4.5 times their original length. Conductivities of about $400 \mathrm{~S} / \mathrm{cm}$ with significantly greater tensile strength $(\sim 145 \mathrm{MPa})$ and crystallinity can be obtained upon doping with $\mathrm{HCl}$ after stretching ${ }^{7}$. This permits it to be solution processed to produce large, flexible, free standing films of the emeraldine base polymer which can be protonated (doped) with a non-oxidising protonic acid such as $\mathrm{HCl}$ to films having conductivities of $\sim 10 \mathrm{~S} / \mathrm{cm}^{16}$. On the basis of conductivity, and stability of the product, $\mathrm{HCl}$ and $\mathrm{H}_{2} \mathrm{SO}_{4}$ are the best dopants for protonation of PANi. The electrochemical polymerisation of aniline is believed to proceed via the cation radical according to the following scheme ${ }^{22}$. It has been found that the growth mechanism of PAni is autocatalytic ${ }^{23}$. It is not due to a simple buildup of layers on the surface like PPy. The oxidation process is accompanied by the insertion of anion to maintain the charge neutrality. Polyaniline has been reported to have the following idealised structure (Figure 1$)^{24}$.

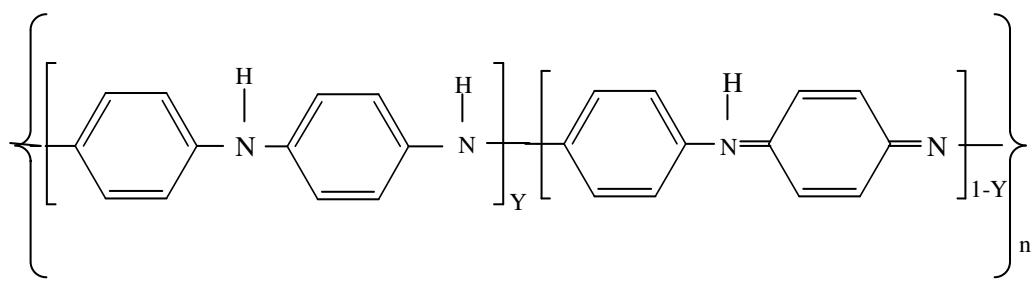

Figure 1 The general structure of PAni conducting polymers 
The structure containing $\mathrm{Y}$ reduced (benzenoid diamine) and (1-Y) oxidised repeat groups(quinoid diamine) where the oxidation state can be defined by the value of (1-Y). Y can in principle be varied continuously from one (the completely reduced material), to zero (the completely oxidised polymer). The completely reduced material $(\mathrm{Y}=1)$ is called Leucoemeraldine and the completely oxidised form $(\mathrm{Y}=0)$ is termed pernigraniline. Intermediate oxidation states where $\mathrm{Y}=0.75$ and 0.25 are called protoemeraldine and nigraniline ${ }^{17}$. The true emeraldine oxidation state $y=0.5$ of polyaniline in which $50 \%$ of the nitrogen atoms are protonated (consists of equal numbers of reduced and oxidised repeat units) is the most highly conducting (metallic) form of the polymer ${ }^{6}$. The relative content of the benzoid and quinoid forms depends on the acid concentration and the degree of oxidation of the polymer ${ }^{22}$. It can be protonated by aqueous acids with a concomitant increase in conductivity of almost 10 orders of magnitude (to a maximum conductivity of $10^{1}-10^{2} \mathrm{~S} \mathrm{~cm}^{-1}$ ), forming a polysemiquinone radical which contains a delocalized half-filled broad polaron energy band ${ }^{25}$. Of the different oxidation states of polyaniline (Figure 2), the emeraldine salt (50\% oxidised) is the only electrically conducting form while all other forms are insulating ${ }^{26,27}$. Each oxidation state can exist in the form of its base or its protonated form (salt) by treatment of the base with acid (Figure 2). These forms may be interconverted by chemical and/or electrochemical oxidation or reduction ${ }^{26}$.

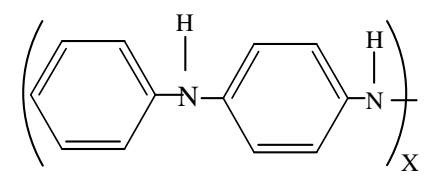

Insulated (reduced form)

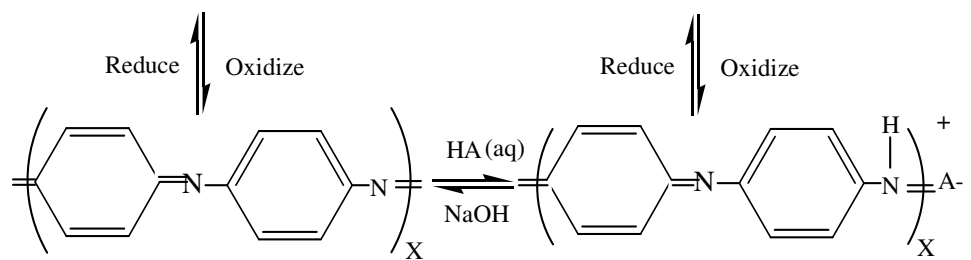

Insulating(oxidized form)

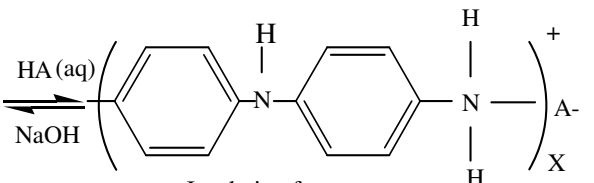

Insulating form

Reduce

Metalic or conducting form

Figure 2 . Four redox forms of polyaniline

\section{Electrical conductivity}

Polyaniline is one of the conducting polymers in which the transition between the insulating and conducting states may involve only migration of a protons a polaron hopping mechanism has been suggested ${ }^{11}$. Cyclic resistometry analysis shows that PAni is less conductive in the fully reduced and fully oxidised states. It is conductive when it is partially oxidised and is the most conductive state when it is 50\% reduced and 50\% oxidised. Electrical conductivity of the polyaniline is strongly sensitive to most synthesis variables and post-treatments ${ }^{12}$. The conductivity of polyaniline, unlike all other conducting polymers depends on two variables, namely the degree of oxidation (electrochemical doping) and the degree of protonation (acid doping) of the material ${ }^{28}$. To a lesser extent, it is also dependent on the type of anion present ${ }^{29}$. However, It has been found that the nature of anion can have an important effect on the properties and molecular structure of the polymer ${ }^{30}$. The conductivity of the electrochemically prepared polyaniline increases with decreasing solution $\mathrm{pH}$ of and polymerisation temperature ${ }^{22}$. 
A maximum conductivity up to $500 \mathrm{~S} / \mathrm{cm}$ at room temperature for oriented PANi films prepared chemically and cast from NMP solvent has been achieved ${ }^{31}$. The conductivity of PANi without doping $(p H 6.3)$ at room temperature is only $10-10 \mathrm{~S} / \mathrm{cm}$. With decreasing $p H$, the conductivity increases with a jump at $p H 3-4{ }^{32}$. It has also been found that moisture acts as a plasticizer and also improves conductivity ${ }^{17}$. It has also been found that drying the polymer under vacuum in order to remove the residual traces of water decreases the conductivity of the polymer while upon exposure to water vapor the conductivity increases ${ }^{33}$.

\section{Electroactivity}

In many applications of polyanilines eg. in batteries ${ }^{37}$ sensors $^{41}$, chemically modified electrode sensors ${ }^{42}$ and $p H$ sensors ${ }^{43}$, membranes for separation ${ }^{44}$ and electrochromic devices $^{45}$, the application relies on the electroactive switching properties of polyanilines. All of these applications require that the material have a highly reversible and rapid switching rate while maintaining chemical stability. The switching properties of polyaniline are affected by the nature of the acid electrolyte ${ }^{46}, p H^{47}$, anions ${ }^{48}$ and the solvent ${ }^{49}$. Polyaniline at $p H$ 6, for example, becomes essentially electrochemically inactive, but regains electroactivity in a completely reversible manner when placed in a more acidic electrolyte ${ }^{8}$. Depending upon the proton content of the polyaniline film and the availability of protons in the medium, one can obtain completely resistive and electroinactive films or electroactive and conducting films ${ }^{49}$. Using cyclic voltammetry $(\mathrm{CV})$ experiments it has been indicated ${ }^{13}$ that the chemically prepared PANi has properties very similar to the electrochemically prepared PANi. The oxidising agent type or concentration has little effect on the electroactivity and the conductivity of the chemically prepared material. The redox reaction of the film is accompanied by colour changes where the film changes from a blue color when the electrode potential is positive of $+0.20 \mathrm{~V}$ to a transparent yellow when it is negative of $0.0 \mathrm{~V}$, and yellow at $-0.20 \mathrm{~V}$ (neutral form) ${ }^{50}$.

\section{Experimental}

A three electrode electrochemical cell was used to prepare PANi conducting polymers used in these studies ${ }^{4}$. Electropolymerisation of polyaniline was carried out using $0.4 \mathrm{M}$ of freshly distilled aniline in a solution of $1 \mathrm{M}$ selected acids. A constant potential $(0.80 \mathrm{~V}$ versus $\mathrm{Ag} / \mathrm{AgCl}$ ) and potentiodynamic method (scanning the potential between -0.20 and $+0.90 \mathrm{~V}$, scan rate $20 \mathrm{mV} / \mathrm{sec}$ ) was employed. Platinium plate was employed as working electrode, platinium (gauze) was used as the auxiliary electrode, and an $\mathrm{Ag} / \mathrm{AgCl}$ in $3 \mathrm{M} \mathrm{NaCl}$ electrode was used as the reference throughout this work. Electroplymerisation was carried out at RT. Polymer powder was removed from the electrode and washed completely with distilled water. The samples dried under ambient conditions before TGA.The chemical polymerization was carried out using freshly distilled aniline and ammonium persulfate as oxidant. The details of the procedure can be found in our previously published work ${ }^{53}$.

\section{Instrumentation}

For the potentiodynamic (CV) growth of PAni, electropolymerisation was carried out using a BAS CV-27. Alternatively, a Princeton Applied Research (PAR) Potentiostat/Galvanostat (Model 363) was employed for potentiostatic deposition of PANi. Thermal Gravimetric Analysis (TGA) was carried out using a Rigaku Thermal Analyzer instrument. The analyses were conducted on $10 \mathrm{mg}$ samples of polymer heated from room temperature to $500^{\circ} \mathrm{C}$. 


\section{Results and Discussion}

Synthesis of Polyaniline conducting polymers

Polyaniline can be synthesised either chemically or electrochemically in aqueous and nonaqueous solvents. The overall polymerisation reaction of PANi can be simply shown in Figure 3.

$\mathrm{n}$<smiles>Nc1ccccc1</smiles>

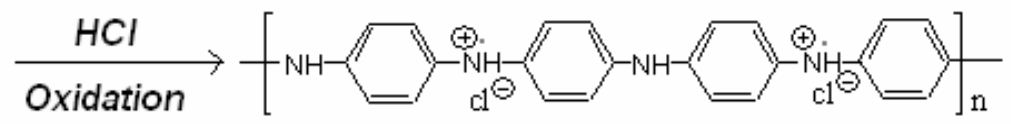

Figure 3 polymerisation reaction of polyaniline

The oxidation process is accompanied by the insertion of anions of acid electrolyte in order to maintain the charge neutrality of the final polymer. However, PANi in contrast to polypyrrole conducting polymers could not be prepared as free standing film electrochemically. Electrochemically prepared PANi has lower conductivity, a powdery appearance and easily wipes off the electrode surface. For electrochemical deposition of $\mathrm{PANi}$, the growth potential of polyaniline was first determined using CV method as shown in Figure 4. Polymer samples were prepared employing the potentiostatic or CV methods because of the greater control which can be achieved over the electrode potential. From the $\mathrm{CV}$ recorded during electrodeposition, it was found that a suitable potential for potentiostatic growth of polymer was found $0.8 \mathrm{~V}$ (see Figure 4). Below this potential, the rate of oxidation was too low. Anodic polarization at potentials more than $0.85 \mathrm{~V}$ causes oxidation leading to gradual degradation of polymer film.

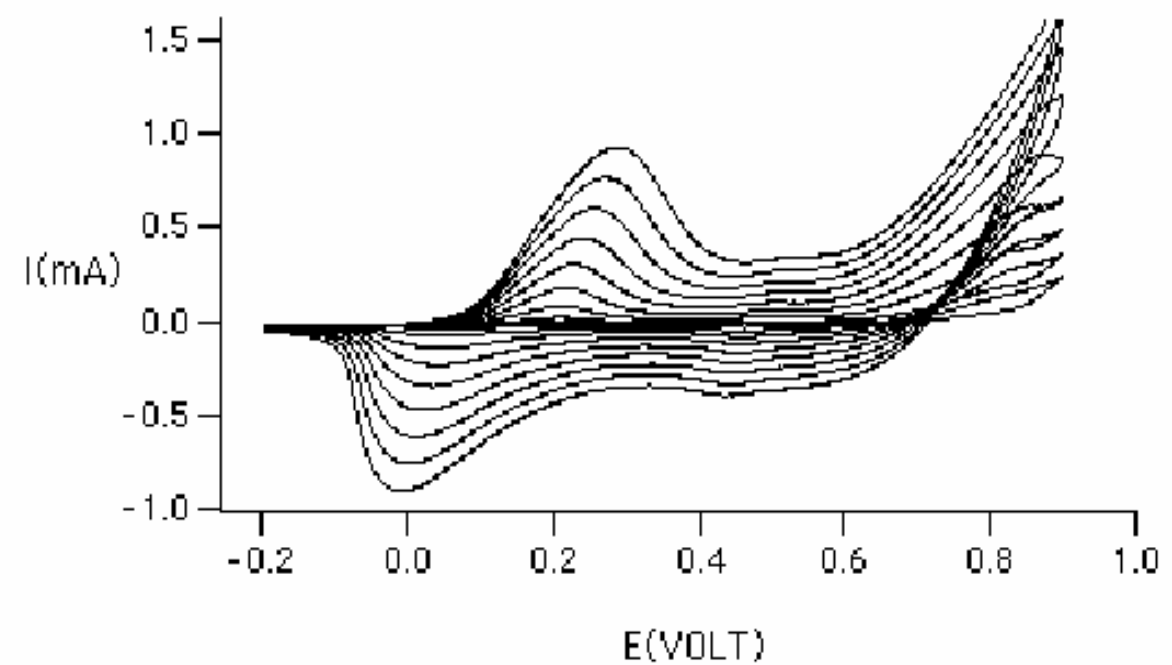

Figure 4. Cyclic voltammograms (CV) recorded during growth for polyaniline.

Scan rate $=20 \mathrm{mV} / \mathrm{sec}$. Electropolymerisation was carried out using a solution of $0.4 \mathrm{M}$ aniline in $1 \mathrm{M} \mathrm{HCl}$. 
A typical CV of a polyaniline sample prepared electrochemically is shown in Figure 5.

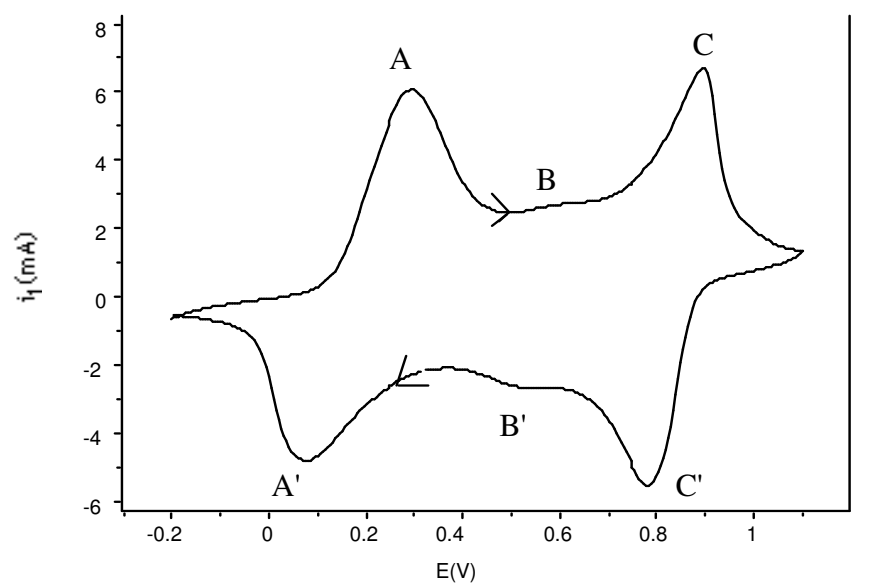

Figure 5. Typical cyclic voltammogram (CV) of Polyaniline synthesised electrochemically (Eapp. $=0.80 \mathrm{~V}$ versus $\mathrm{Ag} / \mathrm{AgCl}$ ) from a solution of $0.4 \mathrm{M}$ aniline and $1 \mathrm{M} \mathrm{HCl}$. CV was recorded after growth in $1 \mathrm{M} \mathrm{HCl}$ as supporting electrolyte. Glassy carbon was used as the working electrode. Scan rate was $20 \mathrm{mV} / \mathrm{s}$.

The responses observed (A/A') and $\left.\mathrm{C} / \mathrm{C}^{\prime}\right)$ are due to the reactions described in the following equations(Figure 6)
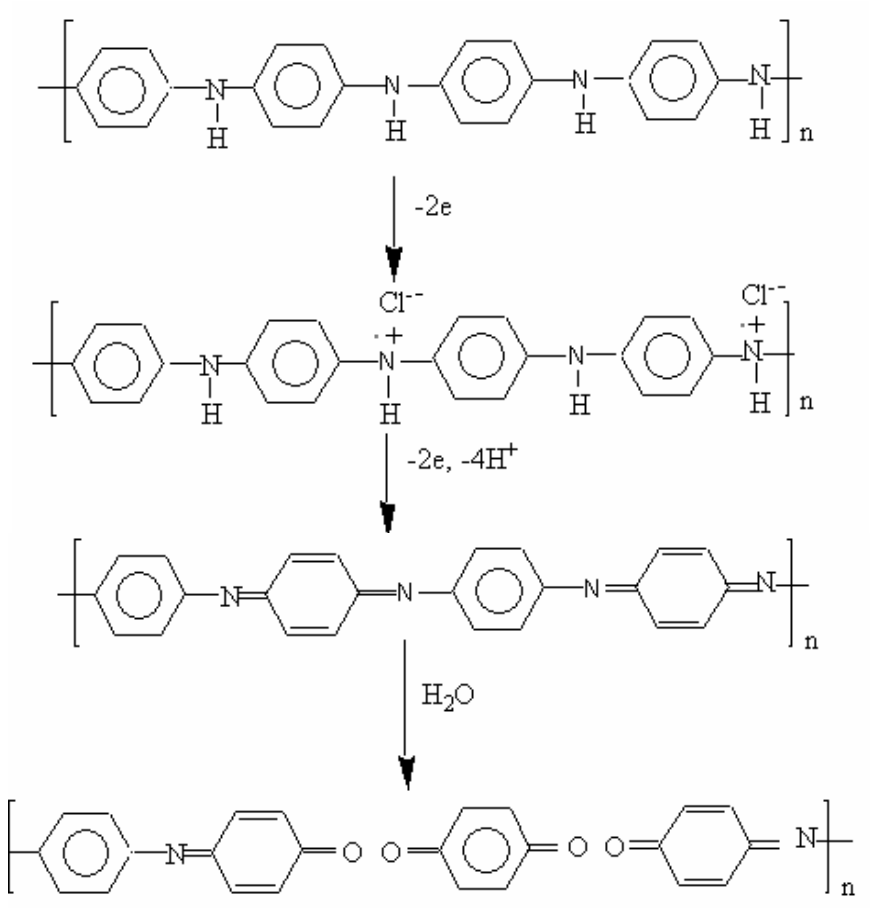

Figure 6. Redox reactions of PANi 
Electroneutrality is maintained by the incorporation of anions from solution. Initial electrochemical responses occur without loss or gain of protons, however, the second electrochemical response involves protonation and deprotonation. The middle peak $\left(\mathrm{B} / \mathrm{B}^{\prime}\right)$ $0.5 \mathrm{~V}$ in the cathodic scan can be related to the formation of quinones (mostly benzoquinone) as a consequence of a hydrolysis reaction in water.

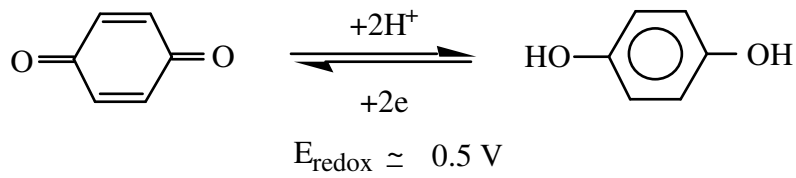

It has been reported that the CV analysis of PANi prepared electrochemically in the presence or absence of aniline suffers from degradation when the potential exceeds $+0.85 \mathrm{~V}$ and below this potential, it is stable and the redox reaction of the film is reversible. This has been attributed to hydrolysis of the imine to form benzoquinone. Upon electrochemical doping, delocalized positive charges form along the PANi chains. Using quartz crystal microbalance (QCM) technique it has been shown ${ }^{51}$ that the loss of electrochemical responses is accompanied by a large frequency increase which indicates that the hydrolysis (changing imine into benzoquinone) products of the oxidised film are soluble and leave the surface. Because of the $p \mathrm{H}$ dependence of the second oxidation process the maximum potential which can be attained without film degradation is also $p \mathrm{H}$ dependent ${ }^{51}$. The rate of electrochemical reversibility of polyaniline prepared in aqueous solution with various acid electrolytes depends on the acidity of the solution but is independent of the nature of counterion ${ }^{52}$. The films are also electroactive in non-aqueous solvents containing both organic salt and protic acid electrolyte.

\section{Effect of thermal treatment on electroactivity of PANi}

We have shown ${ }^{53}$ that the electroactivity of polyaniline starts to decrease after $70^{\circ} \mathrm{C}$ but then shows little further deterioration until $150^{\circ} \mathrm{C}$. This seems to be almost independent of the drying atmosphere, although the inert one is slightly better than air. The electroactivity of polyanilines doped with $\mathrm{HCl}$ prepared chemically or electrochemically both in reduced and oxidized and fully oxidized forms are unstable at $\mathrm{T}>150^{\circ} \mathrm{C}$ in air but keep their electroactivity up to $300{ }^{\circ} \mathrm{C}$ under nitrogen. Since the thermal stability of the polyaniline is greatly dependent on the type of the dopant acid employed during synthesis. By contrast, the exposure to an oxygenated environment at elevated temperatures at $200{ }^{\circ} \mathrm{C}$ resulted in a total loss in electroactivity. It may be concluded that the rate of decay of electroactivity was much faster when samples were heated in air at $\mathrm{T}>150{ }^{\circ} \mathrm{C}$. This is probably due to chemical reaction of $\mathrm{O}_{2}$ with the highly conjugated PAni system. This was not observed when the polymer was exposed to this temperature in an inert atmosphere. A major irreversible loss in electroactivity was observed at $\mathrm{T}>200{ }^{\circ} \mathrm{C}$ in air and $\mathrm{T}>$ $300{ }^{\circ} \mathrm{C}$ in nitrogen ${ }^{53}$. Some changes in polymer structure such as crosslinking ${ }^{35}$ or chlorination ${ }^{38}$ of aromatic rings at high temperature are likely reasons for the loss of electroactivity after thermal treatment under nitrogen at temperatures below the decomposition temperature. In air, however, the chemical reaction of oxygen with the polymer seems to be responsible for the electroactivity decay.

\section{Effect of thermal treatment on conductivity of PANi prepared chemically}

In order to measure conductivity of PANi, the polymer was synthesized chemically and then used solvent cast method ${ }^{53}$, since electrochemically prepared PANi is powdery form. The Effect of thermal treatment on dry state electrical conductivity of polyaniline membranes has been summarized in Table 1 . 
Table 1. Effect of thermal treatment on dry state electrical conductivity of polyaniline membranes prepared chemically and casted from NMP

\begin{tabular}{|c|c|c|c|c|c|c|}
\hline $\mathrm{T}\left({ }^{\circ} \mathrm{C}\right)$ & As grown & 100 & 150 & 200 & 300 & 350 \\
\hline$(\mathrm{S} / \mathrm{cm})$ & $25 \pm 3$ & 0.2 & 0.03 & $\mathrm{i}$ & i & $\mathrm{i}$ \\
\hline$\sigma^{\mathrm{T}}(\mathrm{HCl})(2)(\mathrm{S} / \mathrm{cm})$ & $25 \pm 3$ & $25 \pm 3$ & 20 & 5 & 4 & $\mathrm{i}$ \\
\hline
\end{tabular}

Note: The dimensions of samples prepared $5 \mathrm{~mm}$ (width) $\times 60 \mathrm{~mm}$ (length) $\times 30 \pm 3 \mu \mathrm{m}$ (thickness). The room temperature dry state conductivity of the polymer films in thei doped state $\left(\sigma^{\circ}\right)$ was $25 \pm 3 \mathrm{~S} / \mathrm{cm}$. The other data obtained are the average of three tests for each temperature.

(i) The conductivity was too low to be measured by the 4-point probe method.

(ii) $\sigma^{T}$ is the conductivity of polymer after thermal treatment at temperature $T$

(iii) $\sigma^{T}(\mathrm{HCl})$ is the conductivity after soaking thermally treated polymers in solution of $1 \mathrm{M} \mathrm{HCl}$ for $24 \mathrm{~h}$ at room temperature.

As our data show (Table 1), conductivity decreased after even mild heat treatment and the loss in conductivity observed after thermal treatment up to $100{ }^{\circ} \mathrm{C}$ could be fully recovered after soaking in acid. The loss in conductivity up to $100{ }^{\circ} \mathrm{C}$ is most likely due to simple dehydration of the polymer and evaporation of free dopant, $\mathrm{HCl}$. After treatment at $150{ }^{\circ} \mathrm{C}$ the conductivity could be recovered as much as $80 \%$ by exposing to the acid media. However, thermal treatment at higher temperatures than $150{ }^{\circ} \mathrm{C}$ caused more irreversible loss in dry state conductivity. It seems that exposure of PANi to elevated temperatures (up to about $150-200{ }^{\circ} \mathrm{C}$ ) can have an annealing effect on chemically prepared polyaniline. With thermal treatment under inert atmosphere the regularity of the polymer chains can be increased without any structural changes in the polymer backbone such as ring opening. This is thought to lead to a phase change to a looser packed ordered system. However, in an oxygenated atmosphere a range of reactions are possible including introduction of substitutions along the polymer chains and subsequent loss of conjugation and conductivity. At temperature exceeding $200{ }^{\circ} \mathrm{C}$ conductivity of the polymer decreased rapidly and polymer becomes an insulator.

It has also been reported ${ }^{33-37}$ that the irreversible conductivity decay of thermally treated PANi at temperatures below the thermal decomposition has been attributed to changes in morphology, crosslinking, possible structural rearrangements or other chemical reactions such as chemical interactions between dopant or solvent with the polymer, and irreversible conversion of ionic chlorine to covalent chlorine. Crosslinking reactions and morphological changes may be responsible for decrease in the re-protonation after the thermal ageing.

The conductivity changes observed with exposure to temperatures up to $150{ }^{\circ} \mathrm{C}$ in air were very close to the data obtained in nitrogen. However, at temperatures greater than 150 ${ }^{\circ} \mathrm{C}$ the conductivity decay after treatment in air was more irreversible than after treatment in nitrogen. At higher temperatures, thermal degradation is a predominant factor. The decrease in $\sigma$ is also dependent on sample form, environment, dopant time and temperature of exposure ${ }^{38-40}$. Spectroscopic studies revealed that the doping process of an $\mathrm{HCl}$ - doped film of PANi could be thermally reversed at $180{ }^{\circ} \mathrm{C}$ under $\mathrm{N}_{2}$ without any indication of major changes in the structure of the polymer backbone, but the possibility of substitution of the aromatic rings and cross linking of the polymer chains that could lead to decrease in the conjugation length can not be excluded ${ }^{40}$. Other workers have reported that thermal treatment at up to $150{ }^{\circ} \mathrm{C}$ is favorable to the crystallisation of both doped and undoped PANi 
and heating at more extreme temperatures crystallinity decreases and the polymer becomes more amorphous. X-ray photo electron spectroscopy (XPS) experiments on thermally treated PAni-HCl samples indicate that the irreversible conversion of ionic chlorine to covalent chlorine becomes significant at temperatures greater than $100{ }^{\circ} \mathrm{C}^{33}$.

\section{Thermal Gravimetric Analysis (TGA)}

The technique of TGA has been found one of the most useful techniques for evaluating the onset of thermal decomposition temperature and determining thermal stability of conducting polymers. The TGA results obtained from $\mathrm{PANi}^{35,53}$ show that the method of synthesis is important in determining the thermal stability of the polymer but the oxidation state of the polymer is not very important. A three step decomposition process for the protonated conducting form has been proposed by previous workers. They suggest that the initial stages of weight loss are due to the volatilization of water molecules, then at higher temperatures the protonic acid component of the polymer is lost and finally at more extreme temperatures the degradation of the polymer can lead to production of gases such as acetylene and ammonia. The thermograms (a plot of mass vs. temperature) obtained for $\mathrm{PANi} / \mathrm{HCl}$ prepared by constant potential $(\mathrm{CP})$ and cyclic voltammetry methods $(\mathrm{CV})$ are shown in Figures 5 and 6 respectively.

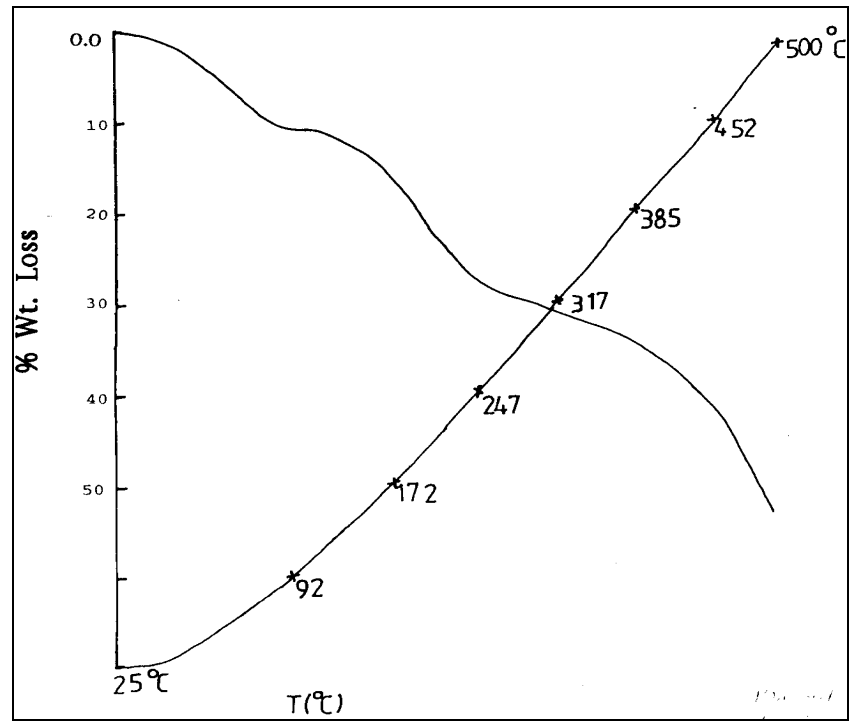

Figure 5. TGA of PAN prepared potentiostatically at $0.8 \mathrm{~V}$. A heating rate of $10{ }^{\circ} \mathrm{C} / \mathrm{min}$ was employed throughout this work unless otherwise stated. The experiments were performed under nitrogen atmosphere. The flow rate of $\mathrm{N}_{2}$ gas over the samples $80 \mathrm{ml} / \mathrm{min}$.

A three step decomposition process for the protonated conducting is observed for both potentiostatic and CV produced acid doped polymers, three separate stages of weight loss were observed under $\mathrm{N}_{2}$. The first continues weight loss that is observed above room temperature may be attributed to loss of moisture, free $\mathrm{HCl}$ and unreacted monomer. The second may be attributed to loss of dopants from deeper sites in the material. At more extreme temperatures (the third weight loss) degradation of the polymer backbone is occurred. 


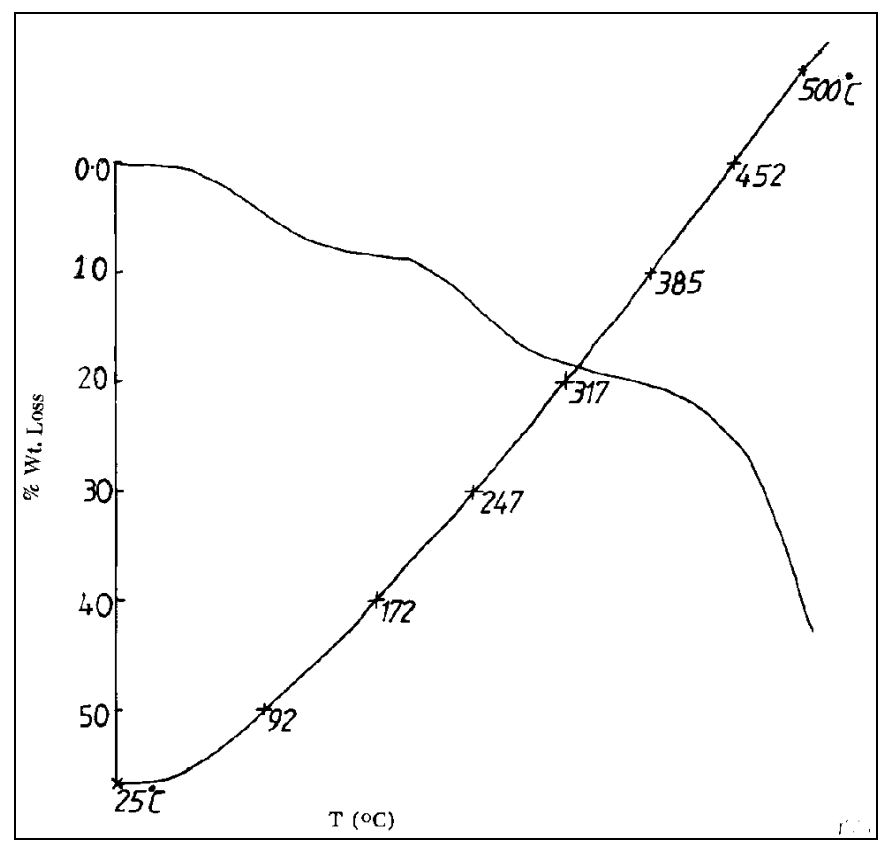

Figure 6. TGA of $\mathrm{PAn} / \mathrm{HCl}$ prepared by $\mathrm{CV}$ method. The $\mathrm{TG}$ analysis conditions were the same as used in Figure 5.

According to our TGA results, the temperature required for degradation were slightly higher for samples prepared using the CV method. It may be concluded that PANi conducting polymers synthesised by CV method is more thermally stable than those prepared by $\mathrm{CP}$ methods. It was also observed that cyclic potential sweep deposition produces more adherent films with higher structural integrity than potentiostatic deposition. As our TGA show, continuous weight loss was observed above room temperature although this may be broken down into three steps. Up to a temperature of $150{ }^{\circ} \mathrm{C}$, may be attributed to evaporation of moisture, unreacted monomer, free $\mathrm{HCl}$ and probably some low molecular weight polymer segments formed during chemical polymerisation. The major component of the volatiles seems to be water molecules. The major weight loss of chemically synthesised $\mathrm{PANi} / \mathrm{HCl}$ at around $200{ }^{\circ} \mathrm{C}$ in the TGA curves was attributed to $\mathrm{HCl}$ loss. The major mass loss occured at $380{ }^{\circ} \mathrm{C}$ is due to polymer backbone degradation. The thermograms (TG) obtained for $\mathrm{PAn} / \mathrm{H}_{2} \mathrm{SO}_{4}$, and PANi/HPTS are shown in Figures 7 and 8.

The initial weight loss observed in TGA of $\mathrm{PANi} / \mathrm{H}_{2} \mathrm{SO}_{4}$ (Figure 7) from 50 to $100{ }^{\circ} \mathrm{C}$ can be attributed to loss of moisture. The higher moisture content of this polymer compared to $\mathrm{PAn} / \mathrm{Cl}$ may be due to the hygroscopic properties of the dopant acid $\left(\mathrm{H}_{2} \mathrm{SO}_{4}\right)$. The larger mass loss (compared to $\mathrm{PAn} / \mathrm{HCl}$ ) occurred at temperatures between 200 and $320{ }^{\circ} \mathrm{C}$. This is most probably due to the evaporation of acid bound to the polymer chain as protonating dopant. PANi/HPTS showed higher thermal stability than PANi doped with $\mathrm{HCl}$ or $\mathrm{H}_{2} \mathrm{SO}_{4}$ acids (see Figure 8). 


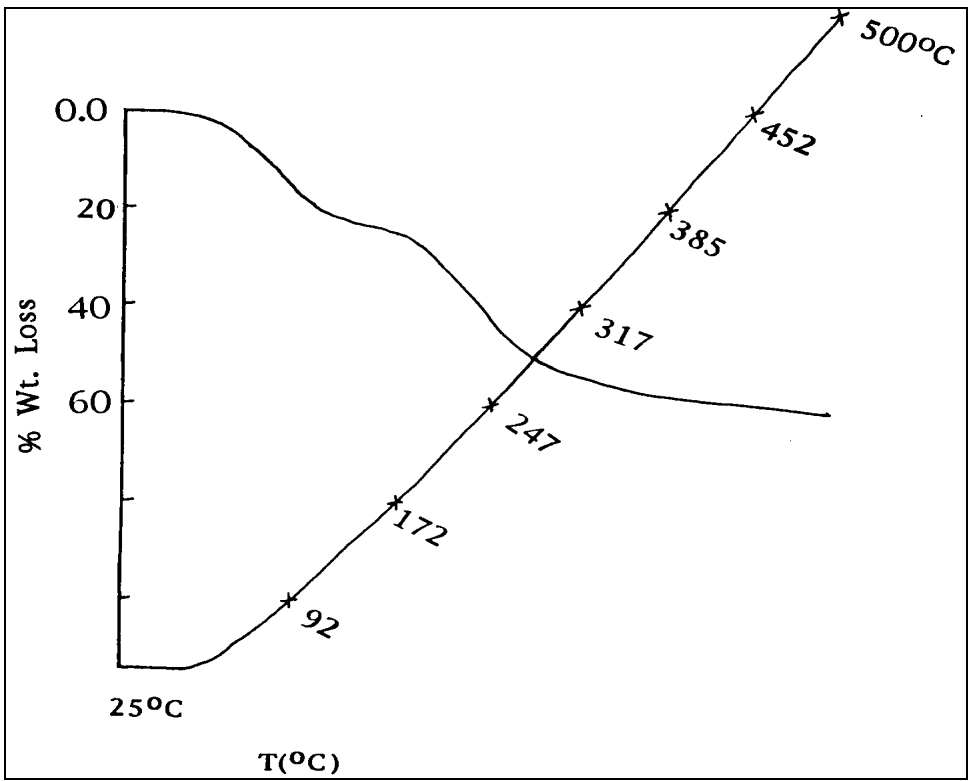

Figure 7 Thermogram of $\mathrm{PANi} / \mathrm{H}_{2} \mathrm{SO}_{4}$. The TGA experimental conditions were the same as used in Figure 5.

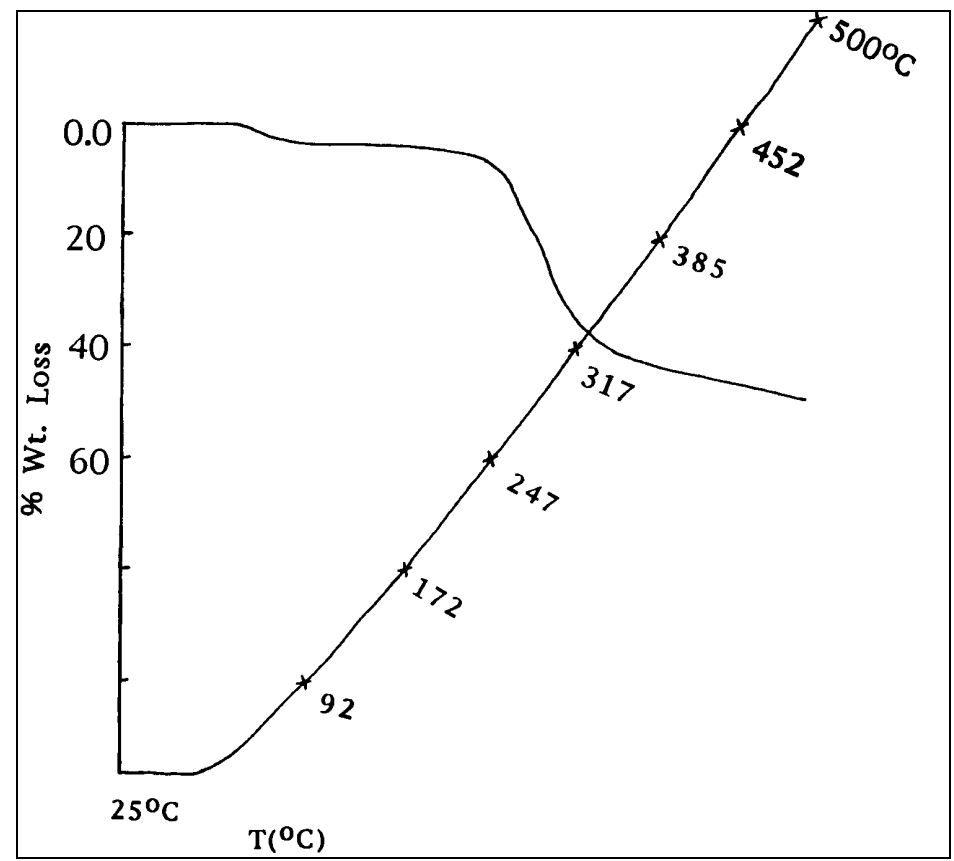

Figure 8. Thermogram of PANi/HPTS. The TGA experimental conditions were the same as used in Figure 5. 
The major weight loss in PANi/HPTS occurred at temperatures between $250{ }^{\circ} \mathrm{C}$ and $320{ }^{\circ} \mathrm{C}$. The initial weight loss observed at $110^{\circ} \mathrm{C}$ may be attributed to solvent evaporation (water). The second weight loss observed at $250{ }^{\circ} \mathrm{C}$ is probably due to the evaporation of the dopant. The polymer backbone seems to be thermally stable up to $500{ }^{\circ} \mathrm{C}$. Differences were observed between polyaniline grown by a sweep potential (CV) method than those grown by constant potential method. Weight losses were greater and more rapid with the latter. This may be due to a lower amount of impurities in the polymer from the CV method. There was little difference between the stability of the reduced and oxidised states of PANi ${ }^{53}$. Thermal stability of electrochemically reduced form of PANi measured by TGA was very similar to the oxidised form of polymer, but showed lower stability than oxidised form, when TGA were carried out in air. It was also found that the doped polymers are significantly less thermally stable than the chemically undoped polyaniline (EB). The thermogram obtained from EB form of polyaniline has been shown in Figure 9. As the TG of EB shows, emeralidine base (EB) powder synthesised chemically is the most thermally stable form of polyaniline (Figure 9).

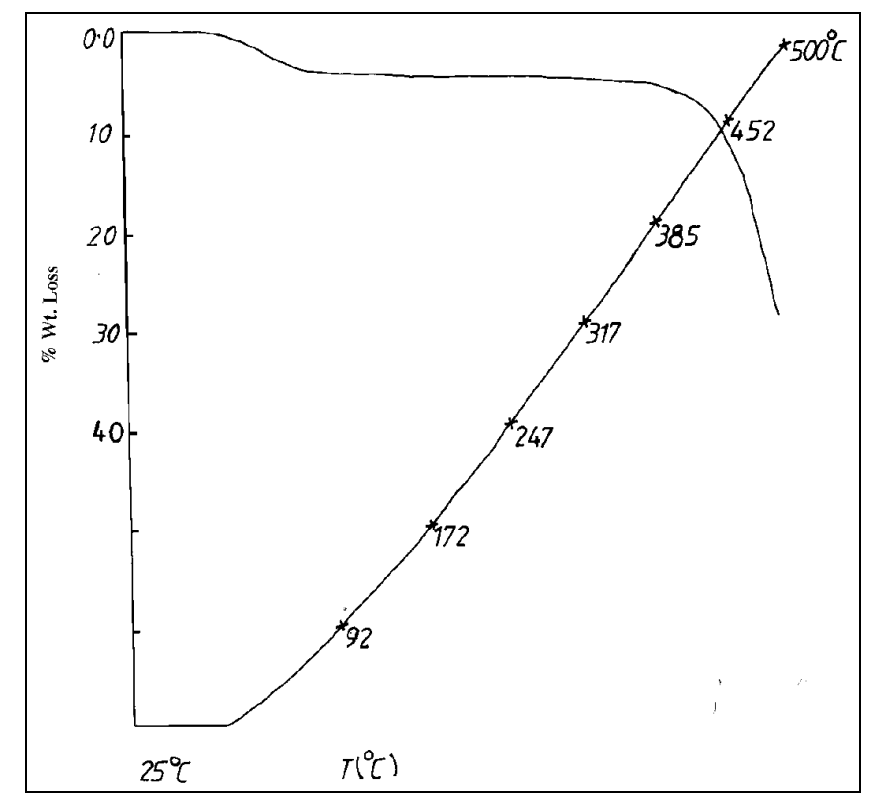

Figure 9. TGA on emeraldine base (EB) powder. The TGA experiment was carried out at using the same conditions given in Figure 5

As our TG analysis show (Figure 9), after the initial weight loss (5\%) observed at $100^{\circ} \mathrm{C}$ minimal mass loss was observed until the temperature was $400{ }^{\circ} \mathrm{C}$. The major weight loss was observed at $420{ }^{\circ} \mathrm{C}$. The total weight loss at $\mathrm{T}=500{ }^{\circ} \mathrm{C}$ was $29 \%$. After $600{ }^{\circ} \mathrm{C}$ polymer decomposition is almost complete. TGA profiles confirmed that the polymer material was stable to at least $300{ }^{\circ} \mathrm{C}$. Emeralidine base (EB) form of polyaniline was found to be the most thermally stable form of polyaniline. TGA results indicated ${ }^{57}$ that undoped polyaniline samples (EB) show lower weight losses before the thermal breakdown of the polymer backbone and thermal stability of the intrinsically oxidized PANi is dependent to dopant acid and is generally poor. 
It has been reported ${ }^{59,60}$ that longer heat treatment time of PANi-EB leads to more pronounced cross-linking which in turn lead to reduced solubility of the polymer and to reduced conductivity recovering after post treatment in acids. This originates in fewer imine $\mathrm{N}$ atoms being available for reprotonation. From TGA, IR spectroscopy and elemental analysis, it has been found that in air, oxidation and possible structural rearrangements lead to loss in conductivity (because of loss in conjugation, not dopant) at temperatures below thermal decomposition ${ }^{34}$. It was also noted that the structural integrity of doped PANi, as probed by infrared spectroscopy, was maintained during thermal ageing at temperatures at or below $200^{\circ} \mathrm{C}^{35}$. Neoh et al..$^{33}$ have reported that when $\mathrm{PANi} / \mathrm{Cl}$ is heated in air at $150{ }^{\circ} \mathrm{C}$, the total $\mathrm{Cl}$ content changes little but it is transformed to a deprotonated structure accompanied by $\mathrm{Cl}$ substitution at the aromatic ring. The TG data obtained in air or $\mathrm{N}_{2}$ is consistent with the theory that the formation of covalent chlorine makes it more stable to heat above $250{ }^{\circ} \mathrm{C}$ than the mainly ionically bonded chlorine present in the protonated structure. The rapid weight loss observed in air for temperatures greater than $350{ }^{\circ} \mathrm{C}$ is due to formation of volatile products resulting from the oxidation of the polymer. Thermal stability of polyaniline conducting polymers is also dependent upon some important factors such as, acid dopant, oxidation state of the polymer, monomer substitutions, atmosphere of heat treatment, exposure time and preparation conditions. PANi-EB is the most stable among polyaniline.

\section{Crystallinity of PANi}

It has been found that doped PANi is remarkably crystalline. It becomes almost amorphous when it is undoped with $\mathrm{NH}_{4} \mathrm{OH}$. Heat treatment at up to $150^{\circ} \mathrm{C}$ is favourable to the crystallisation of both doped and undoped PANi. The structure of annealed PANi up to $150^{\circ} \mathrm{C}$ becomes more regular and ordered ${ }^{54}$. Heating at higher temperatures destroys the crystalline structure. Elemental analysis and IR spectroscopy of initial and $150{ }^{\circ} \mathrm{C}$ annealed PANi (under $\mathrm{N}_{2}$ ) showed no significant differences ${ }^{39}$. In this study, X-ray diffraction analysis also supported the increasing regularisation and crystallinity of PANi annealed at $150{ }^{\circ} \mathrm{C}$.

It is interesting to note that the weight loss of the re-doped samples could be lower than the unaged polymer, which might be responsible for the incomplete recovery of the conductivities by re-doping. Therefore, there must be other processes involved during the thermal ageing below and at $200{ }^{\circ} \mathrm{C}$ besides the loss of water and $\mathrm{HCl}$. Wei et al. ${ }^{35}$ suggested that crosslinking reactions along with the morphological changes could take place during the thermal aging. The aged polymer samples at 100 and $200^{\circ} \mathrm{C}$ were also found to have lower solubility than the unaged ones in NMP. Wang and Rubner ${ }^{55}$ obtained spectroscopic evidence which suggests that in the case of polyaniline thermal dedoping occurs due to disordering of the polymer backbone through chemical modifications (substitution of aromatic rings/crosslinking) or changes in molecular reorganisation (thermal disruption of crystalline or paracrystalline regions). They also point out, however, that even spectroscopic studies are limited in that minor structural changes would not be detected and yet these could have a major impact on physical properties. In the later case, the thermal disruption of crystalline or polycrystalline regions of the film could disrupt the ability of the backbone electrons to achieve a high level of delocalisation. In addition, they emphasize the importance of film thickness and the form of the sample (Powder, pressed pellet) on thermal properties, although spectroscopy can only be carried out on thin films. Mattes et al. ${ }^{56}$ also reported that thermal treatment of polyaniline films at $125{ }^{\circ} \mathrm{C}$ for three hours makes the polymer a very dense material which decreases its permeability and increases its selectivity 
for gas separations. Therefore, thermal treatment may also be used to modify the properties of conducting polymers make them desirable for some specific applications. Previous workers ${ }^{39}$ have reported that thermal treatment at up to $150{ }^{\circ} \mathrm{C}$ is favourable to the crystallisation of both doped and undoped PANi and heating at more extreme temperatures crystallinity decreases and the polymer becomes more amorphous. These previous observations are in good agreement with the data reported above. We have also reported ${ }^{53}$; showing an increase in tensile strength after heating, confirm that cross linking reactions occur in the course of thermal treatment.

\section{Environmental stability of PANi conducting polymers}

Polyaniline conducting polymers the same as other reactive and conducting organic materials suffer from their poor thermal stability in air because of the chemical reaction of oxygen with the highly conjugated system of PANi. In contrast to most conducting polymers which are less stable in humid environments, polyanilines are very stable. This clearly indicates that the conductivity stability of PANi is enhanced in the presence of humid air. It was also reported that the water vapour in the air appeared to be an essential factor in the recovery process of conductivity since the sample cooled in the dessicator shows only minimal recovery. It has been reported ${ }^{48}$ that prolonged storage of polyaniline in water or working electrolyte (without polarisation) leads to an irreversible decrease of its electrochemical activity, but when stored dry, its activity is preserved. Polyaniline can be left standing in air and the electroactive properties are not destroyed. Storage in air, however, does alter the film and produces an anodic shift in the peak positions ${ }^{19}$. Pasquali et $a l .{ }^{10}$ have indicated that after storage of polyaniline in distilled water (open circuit) a hydrolysis reaction as evidenced by the appearance of the anodic peak at $\sim 0.5 \mathrm{~V}$ occurs. Degradation of the polymer was higher when stored in a solution of $1 \mathrm{M} \mathrm{H}_{2} \mathrm{SO}_{4}$ (even after one day). The dissolved $\mathrm{O}_{2}$ in $\mathrm{H}_{2} \mathrm{O}$ and impurities contained in the film can favour the deprotonation of polymer, then the rapid degradation.

\section{Conclusions}

Bulk preparation of polyaniline conducting electroactive polymers powder can be achieved using simple chemical method without need to any special equipment. Highly conducting coating or polymer membranes (PANi or composites) with high mechanical properties of polyaniline can be prepared using solvent cast method. The electrical properties, thermal and environmental stability of PANi Films is highly dependent on the nature and the size of its dopant acid. The thermal stability of the acid doped PANi was in the order of PANi/HPTS> $\mathrm{PANi} / \mathrm{H}_{2} \mathrm{SO}_{4}>\mathrm{PANi} / \mathrm{HCl}$. The undoped form of PANi (EB) is the most soluble and more thermally stable than the other forms of PANi. The loss in conductivity and electroactivity of PANi conducting polymers are mostly reversible when thermally treated below $150^{\circ} \mathrm{C}$ in air or nitrogen atmospheres.

\section{References}

1. Lux F, Hinrichsen G, and Pohl M M, J. Polymer Sci. Part B, Polymer Physics, 1994, 32,

2. Andreatto A, Cao Y, Chiang J C, Heeger A J and Smith P, Synthetic Metals, 1988, 26, 383- 389 .

3. Olmedo L, Hourquebie P, and Jousse F, Adv. Mater, 1993, 5, No. 5.

4. Chiang J C and Macdiarmid A G, Synthetic Metals, 1986, 13, 193-205.

5. Wei Y and Hsueh K F, J. Polymer Science: Part A. 
6. MacDiarmid Chang A G, Richter J C, Somasiri A F, and Epstien A J, in "Conducting polymers" (Ed.L. Alcacer), Reidel Publishing Co. Dordrecht. 1987, pp. 105,

7. MacDiarmid A G, Manouar S K, Scherr E M and Xun Tang, Druy M A and Gatkowski P J, Epstein A J, J. Polymer Material Science and Eng., 1991, 64, 254-255.

8. Huang W S, Humphrey B D and MacDiarmid A G, Chem. Soc. Faraday Trans., 1986, 82, 2385-2400.

9. Chan H S O, Ng S C, Seow S H, Sim W S and Hor T S A, J. Thermal Analysis, 1993, 39, 177-185.

10. Pasquali M, Pistoia G and Rosati R, Synthetic Metals, 1990, 53, 1-15.

11. Choi K M, Kim K H and Choi J S, J. Phys.Chem., 1989, 93, 4659-4664.

12. Cao Y, Anreatta A, Heegerand A J, Smith P, Polymer, 1989, 30, 2305-2311.

13. Rodrigues M A and Paoli M A D, Synthetic Metals, 1991, 41, 2957-2962.

14. Gholamian M and Contractor A Q, J. Electroanal. Chem., 1988, 252 291-301.

15. Angelopoulos M, Austurias G E, Ermer S P, Ray A, Mol. Cryst., 1988, 160, 151-163.

16. Wei Y, Jang G W, Hsueh K F, Scherr E M, MacDiarmid A G and Epstein A J, Polymer, 1992, 33, 314-319.

17. Angelopoulos M, Rarnd A, MacDiarmid A G, Epstein A J, Synthetic Metals, 1987, 21, 21-30.

18. Neoh K G, Tan K L, Tan T C and Kang E T, J. Macromol. Sci-Chem, 1990, A27 (3), 347-360.

19. Diaz A F and Logan J A, J. Electroanal. Chem., 1980, 111, 111-114.

20. Cui C Q, Ong L H, Tan T C and Jim Y Lee, Synthetic Metals, 1993, 58, 147-160.

21. Patil S F, Bedaker A G, Agastie C, Mat. Lett., 1992, 14, 307-312.

22. Bhadani S N, Gupta M K, and Gupta S K S, J. Applied Polymer Sci., 1993, 49, 397-403.

23. Stilwell D E, Park S M, J. Electrochem. Soc., 1988, 135, (9), 2254-2262.

24. MacDiarmid A G and Austurias G E, Polymer Reprint, 1989, 30, 147-149.

25. MacDiarmid A G and Epstein A J, Faraday Discuss. Chem. Soc, 1989, 88, 317- 332.

26. MacDiarmid A G, Chiang J C, Halpern M, Mu H. W S, Somasiri S L, Wu N L D, Yaninger W S I, Mol. Cryst. Liq. Cryst., 1985, 121, 173-180,

27. MacDiarmid A G, Yang L S, Huangand W S, Humphrey B D, Synthetic Metals, 1987, 18, 393-398.

28. Chiang J C and Macdiarmid A G, Synthetic Metals, 1986, 13, 193-205.

29. Focke W W, Wnek G E, and Wei Y, J.Physical Chemistry, 1987, 91, 5813- 5818.

30. Majidi M R, Kane- Maguire L A P and Wallace G G, Polymer, 1994, 35, 3113-3115.

31. Wan M, Li M, Li J, and Liu Z, J. Applied Polymer Sc, 1994, 53, 131-139.

32. Pingsheng H, Xiaohua Q and Chune L, Synthetic Metals, 1993, 55-57, 5008-5013.

33. Neoh K G, Kang E T, Khor S H and Tan K I, Polymer Degradation and Stability, 1990,27, 107-117.

34. Kulkarni V G and Mathew W R, Synthetic Metals, 1991, 41-43, 1009-1012.

35. Wei Y and Hsueh K F, J. Polymer Science: Part A: Polymer chemistry, 1989, 27, 4351-4363.

36. Kulkarni V G, Thermochimica Acta, 1991, 188, 265-272.

37. Macdiarmid A G, Yang L S, Synthetic Metals, 1987, 18, 393-398.

38. Hagiwara T, Yamaura M and Iwata K, Synthetic Metals, 1988, 25, 243-252.

39. Fossong W, Jinsang T, Lixiang W, Hong Fang Z and Zhishen M, Mol. Cryst. , Liq. Cryst., 1988, 160, 175-184. 
40. Kulkarni V G, Cambell L D and Mathew W R, Synthetic Metals, 1989, 30, 321-325.

41. Wang E and Liv A, J Analytica Chemica Acta, 1991, 252, 53 - 57.

42. Ge H and Wallace G G, Polymer, 1992, 33, 2348-2352.

43. Granstrom M and Inganas O, Synthetic Metals, 1992, 48, 21-31.

44. Mirmohseni A, Price W E, Wallace G G and Zhao H, J. Intelligent Material Systems and Structures, 1993, 4, 43-49.

45. Morita M, J. MaKromol. Chem. 1993, 194, 1513-1526.

46. Wang B, Tang J and Fossong wang, Synthetic Metals, 1986, 13, 329-334.

47. Rudzinski W E, Lozano L, and Walker M, J. Electrochem. Soc., 1990, 137 (10).

48. Kazarinov V E, Andreev V N, Spytsin M A and Shlepakov A V, Electrochemica Acta, 1990, 35, 899-904.

49. Pekmez N, Pekmez K and Yildiz A, J. Electroanalytical Chemistry, 1994, 370, 223-229.

50. Kobayashi T, yoneyama H and Tamura H, J. Electroanal. Chem., 1984,161, 419-423.

51. Orata D and Buttry D A, J. Am. Chem. Soc., 1987, 109, 3574-3581.

52. LaCroix J C and Diaz A F, J. Electrochemical Soc., 1988,135, 1460 - 1463.

53. Ansari R, Price W E and Wallace G G, Polymer, 1996, 37, 917-923.

54. Atsushi K, Hitoshi I, Kosuke A, Masaharu S, J. Appl. Phys. 1993, 74, 296-299.

55. Wang Y and Rubner M F, Synthetic Metals, 1992, 47, 255-266.

56. Mattes B R, Anderson M R, Conklin J A, Reiss H, and Kaner R B, Synthetic Metals, 1993, 55-57, 3655-3660.

57. Rong Zeng X and Man Ko Tze, Polymer, 1998, 39, 1187-1195.

58. Cataldo F and Paolo Maltese, European Polymer Journal, 2002, 38, 1791-1803.

59. Chandrayanthi N and Careem M T, Polymer Bulletin, 2000, 44, 101-108.

60. Wenguang Li and Meixang Wan, J. Appl. Polym. Sci, 1999, 71, 615-621. 


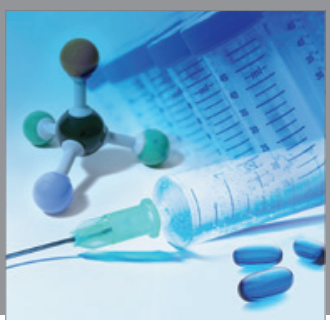

International Journal of

Medicinal Chemistry

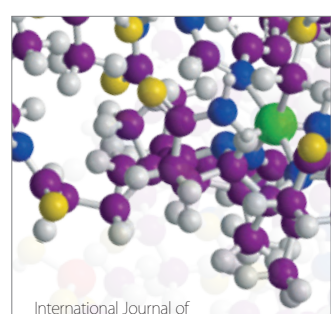

Carbohydrate Chemistry

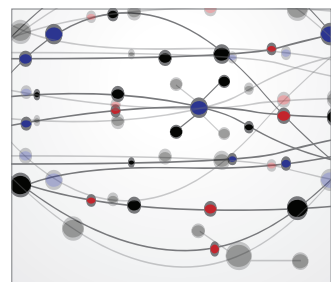

The Scientific World Journal
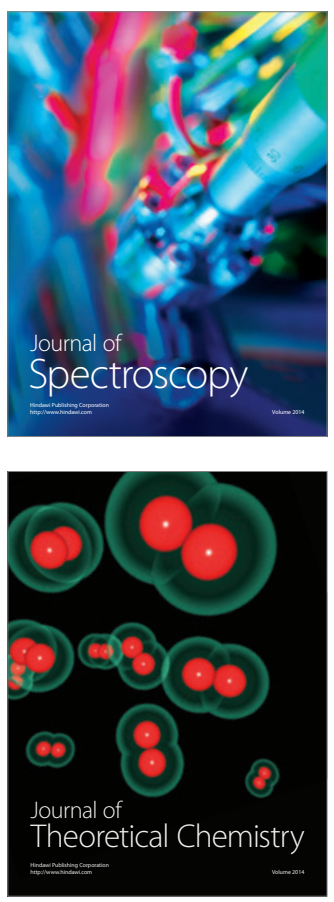
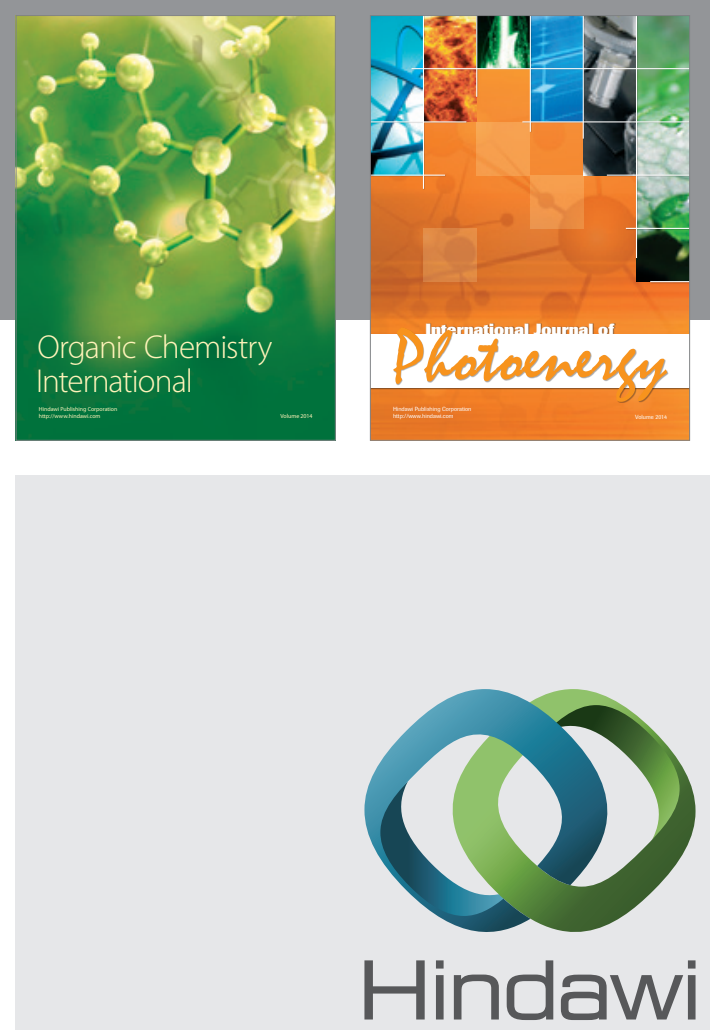

Submit your manuscripts at

http://www.hindawi.com
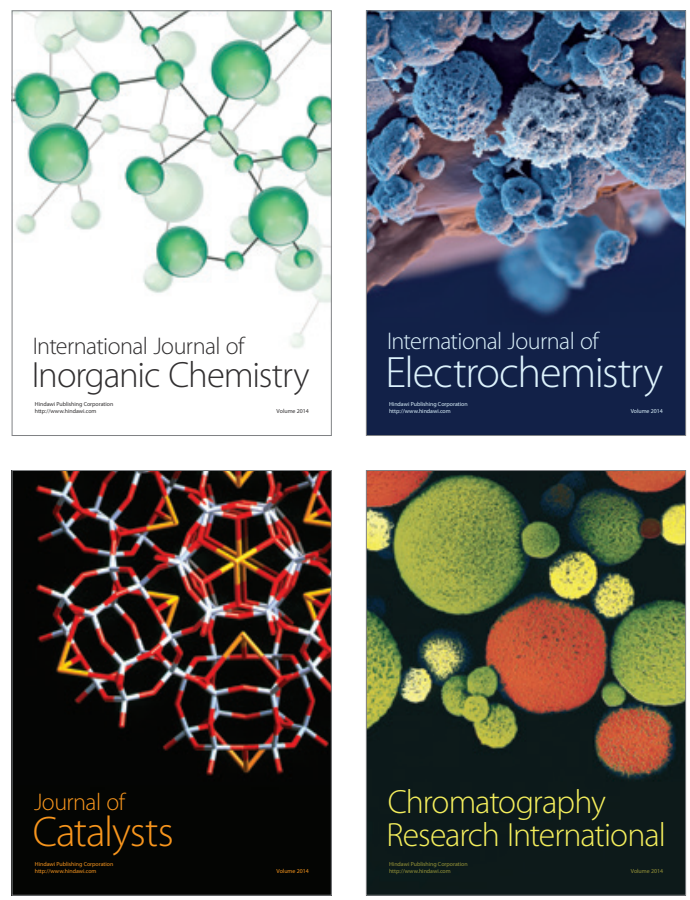
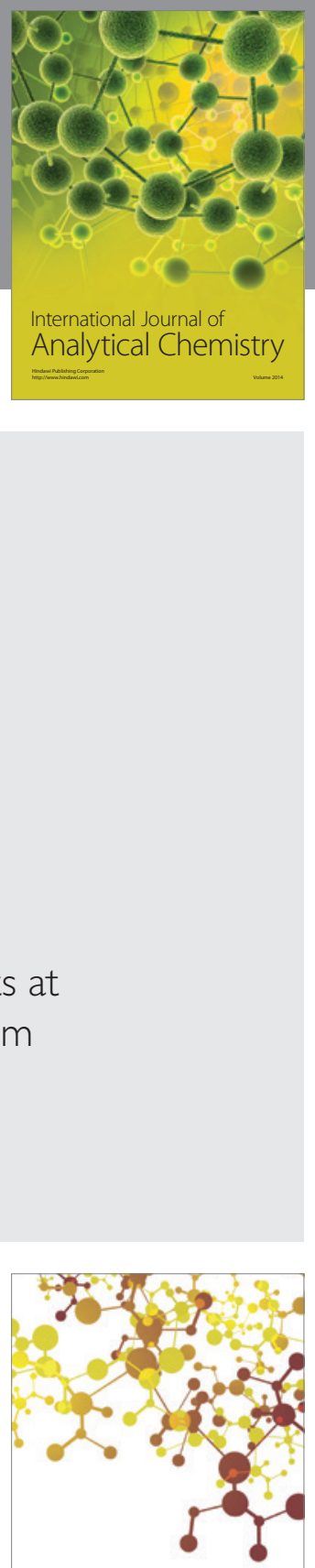

Journal of

Applied Chemistry
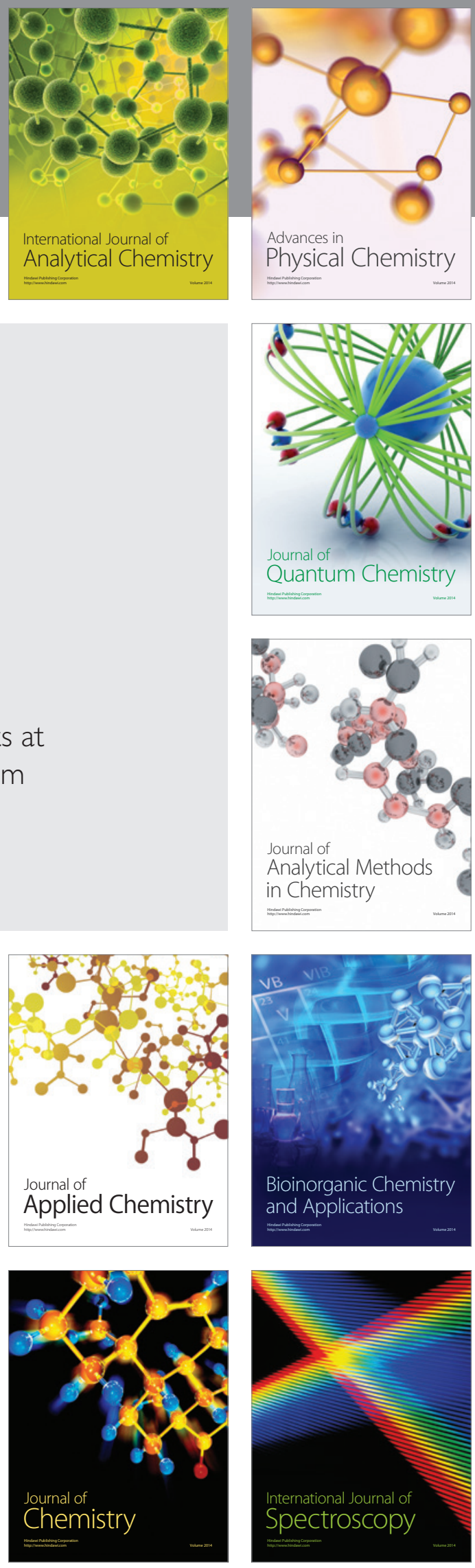